PROCEEDINGS OF THE

AMERICAN MATHEMATICAL SOCIETY

Volume 125, Number 5, May 1997, Pages 1413-1417

S 0002-9939(97)03699-X

\title{
ON A NEGATIVE RESULT CONCERNING INTERPOLATION WITH CHANGE OF MEASURES FOR LORENTZ SPACES
}

\author{
ELIDA V. FERREYRA
}

(Communicated by J. Marshall Ash)

\begin{abstract}
In this note we show that the Stein-Weiss theorem on $L^{p}$ interpolation with change of measures cannot be extended to Lorentz spaces $L^{p, s}$.
\end{abstract}

By the well-known Marcinkiewicz interpolation theorem, the $L^{p}$ boundedness of a sublinear operator for all $p$ in some open interval $\left(p_{1}, p_{2}\right)$ follows from some weak type inequalities for the extreme values $p_{1}$ and $p_{2}$. These inequalities can be viewed as boundedness properties on some appropiate Lorentz spaces, and, in fact, the theorem admits an extension to such spaces (see Theorem 1 below).

In 1958, Stein and Weiss proved an interpolation result on $L^{p}$ spaces which allows one to change measures simultaneously with changing exponents. (A particular case is stated below as Theorem 2). It is natural to ask whether it is possible to obtain an extension of their result in the context of Lorentz spaces. In this note we prove that the answer is negative.

In what follows we first give some definitions; next we state some known interpolation results and present the conjecture about interpolation with change of measures on Lorentz spaces. Finally we state and prove two lemmas establishing that the conjecture is false.

The fact that this conjecture is false is a stumbling stone in the study of oneweight norm inequalities for the fractional maximal operator on Lorentz spaces. In fact, about the one-weight weak inequalities of Muckenhoupt and Wheeden [M-W] for such an operator it is only known that they extend to Lorentz spaces $L^{p, s}$ when the range of $\mathrm{s}$ is restricted. More precisely, the author obtained in $[\mathrm{F}]$ the following result.

Let

$$
M_{\alpha} f(x)=\sup \frac{1}{|Q|^{1-\alpha / n}} \int_{Q}|f(y)| d y,
$$

where $0<\alpha<n$ and the sup is taken over all cubes $Q$ with center at $x$. Assume $p, q$ and $s$ are such that $1 / q=1 / p-\alpha / n$ and that either $1<p<n / \alpha, 1<s \leq q$ or $p=s=1$. Then there exists a constant $C$, independent of $f$, such that

$$
\left\|M_{\alpha} f\right\|_{q, \infty, w^{q}} \leq C\|f\|_{p, s, w^{p}}
$$

Received by the editors May 25, 1995 and, in revised form, November 16, 1995.

1991 Mathematics Subject Classification. Primary 46B70; Secondary 46E30.

Key words and phrases. Interpolation, Lorentz spaces.

Partially supported by CONICET, CONICOR, SECYT (UNC), Argentina.

(c)1997 American Mathematical Society 
if and only if $w$ satisfies

$$
\left(\frac{1}{|Q|} \int_{Q}[w(x)]^{q} d x\right)^{1 / q}\left(\frac{1}{|Q|} \int_{Q}[w(x)]^{-p^{\prime}} d x\right)^{1 / p^{\prime}} \leq K
$$

for some constant $K$ independent of $Q$.

Let $w$ be a positive weight function defined on $\Re^{n}$. The weighted Lorentz space $L^{p, s}(w)$ is defined as the set of all measurable functions $f$ defined on $\Re^{n}$ such that $\|f\|_{p, s, w}<\infty$, where

$$
\|f\|_{p, s, w}= \begin{cases}{\left[\frac{s}{p} \int_{0}^{\infty} t^{s / p-1} f^{*}(t)^{s} d t\right]^{1 / s},} & 1 \leq p, s<\infty \\ \sup _{t>0} t^{1 / p} f^{*}(t), & 1 \leq p \leq \infty, s=\infty\end{cases}
$$

or equivalently (cf. [Sa])

$$
\|f\|_{p, s, w}= \begin{cases}{\left[s \int_{0}^{\infty} y^{s-1} \lambda_{f}(y)^{s / p} d y\right]^{1 / s},} & 1 \leq p, s<\infty, \\ \sup _{y>0} y \lambda_{f}(y)^{1 / p}, & 1 \leq p<\infty, s=\infty .\end{cases}
$$

Here

$$
\lambda_{f}(y)=w\left(\left\{x \in \Re^{n}:|f(x)|>y\right\}\right)
$$

and

$$
f^{*}(t)=\inf \left\{y>0: \lambda_{f}(y) \leq t\right\}
$$

Therefore, $L^{p, p}(w)$ is the Lebesgue space $L^{p}(w)$. Given a Lebesgue measurable set $E,|E|$ will denote its Lebesgue measure. As usual we take $1 / p+1 / p^{\prime}=1$. We will also use the following duality result which can be found in the literature (see e.g. [C-H-K], or $[\mathrm{O}])$.

There exists a constant $C>0$ such that

$$
C^{-1}\|f\|_{p, s, w} \leq \sup \left\{\left|\int_{\Re^{n}} f g w d x\right|:\|g\|_{p^{\prime}, s^{\prime}, w} \leq 1\right\} \leq C\|f\|_{p, s, w},
$$

for all $f$, where $s=1$ if $p=1$, and $s=\infty$ if $p=\infty$.

We state two interpolation theorems which can be found in the literature. As we mentioned above, Theorem 1 extends the Marcinkiewicz interpolation theorem ([S-W2], pp. 184-185) to Lorentz spaces (see e.g. [B-S], p. 225). Theorem 2 deals with a particular case of the interpolation theorem on $L^{p}$ spaces with change of measures proved by Stein and Weiss in [S-W1].

Theorem 1. Suppose that $1 \leq p_{0}<p_{1}<\infty$ and $1<q_{0}, q_{1}<\infty$ with $q_{0} \neq q_{1}$. If $T$ is a sublinear operator satisfying

$$
\|T f\|_{q_{i}, \infty, w} \leq C_{i}\|f\|_{p_{i}, 1, v},
$$

for all $f \in L^{p_{i}, 1}(v), i=0,1$, then for each $0<t<1$ and $1 \leq s \leq \infty$ there exists a constant $C>0$ such that

$$
\|T f\|_{q_{t}, s, w} \leq C\|f\|_{p_{t}, s, v},
$$

for all $f \in L^{p_{t}, s}(v)$, provided that

$$
\frac{1}{p_{t}}=\frac{1-t}{p_{0}}+\frac{t}{p_{1}} \quad \text { and } \quad \frac{1}{q_{t}}=\frac{1-t}{q_{0}}+\frac{t}{q_{1}} .
$$


Theorem 2. Suppose that $1 \leq p_{0}, p_{1}<\infty, 1<q_{0}, q_{1} \leq \infty$ and $0<r_{0}, r_{1}<\infty$ with $p_{0} \neq p_{1}, q_{0} \neq q_{1}$ and $p_{i} \leq q_{i}$. If $T$ is a sublinear operator satisfying

$$
\|T f\|_{q_{i}, \infty, w} \leq C_{i}\|f\|_{p_{i}, v^{r_{i}}},
$$

for all $f \in L^{p_{i}}\left(v^{r_{i}}\right), i=0,1$, then for each $0<t<1$ there exists a constant $C>0$ such that

$$
\|T f\|_{q_{t}, w} \leq C\|f\|_{p_{t}, v^{r_{t}}},
$$

for all $f \in L^{p_{t}}\left(v^{r_{t}}\right)$, provided that

$$
\frac{1}{p_{t}}=\frac{1-t}{p_{0}}+\frac{t}{p_{1}}, \frac{1}{q_{t}}=\frac{1-t}{q_{0}}+\frac{t}{q_{1}} \quad \text { and } \quad r_{t}=(1-t) r_{0}\left(\frac{p_{t}}{p_{0}}\right)+\operatorname{tr}_{1}\left(\frac{p_{t}}{p_{1}}\right) .
$$

It is natural to conjecture that there is an extension of Theorem 2 that parallels the fashion in which Theorem 1 extends the Marcinkiewicz interpolation theorem.

Conjecture 1. Let $T, p_{i}, q_{i}$, and $r_{i}, i=0,1$, satisfy the hypothesis of Theorem 2. Then for each $0<t<1$ and $1 \leq s \leq \infty$ there exists a constant $C>0$ such that

$$
\|T f\|_{q_{t}, s, w} \leq C\|f\|_{p_{t}, s, v^{r_{t}}},
$$

for all $f \in L^{p_{t}, s}\left(v^{r_{t}}\right)$ and $p_{t}, q_{t}, r_{t}$ as in Theorem 2.

The following lemmas show that this conjecture is false.

The first lemma reduces the conjectured interpolation result to the boundedness of a multiplication operator on Lorentz spaces. The second lemma proves that such boundedness holds only on $L^{p}$ spaces.

Lemma 1. Let $p_{i}, q_{i}, r_{i}, i=0,1$, and $p_{t}, q_{t}, r_{t}$ be as in Theorem 2. Let $\alpha=$ $\left(r_{1}-r_{0}\right) /\left(p_{1}-p_{0}\right)$ and $\beta=\left(r_{0} p_{1}-r_{1} p_{0}\right) /\left(p_{1}-p_{0}\right)$. Define the operator $U$ as

$$
U(f)=v^{\alpha} f .
$$

Then Conjecture 1 is true if and only if the operator $U$ satisfies the inequality

$$
\|U f\|_{p_{t}, s, v^{\beta}} \leq C_{t, s}\|f\|_{p_{t}, s, v^{r_{t}}}
$$

for all $f \in L^{p_{t}, s}\left(v^{r_{t}}\right), 0<t<1$ and $1 \leq s \leq \infty$.

Proof. First we notice that

$$
\|U f\|_{p_{i}, v^{\beta}}=\|f\|_{p_{i}, v^{r_{i}}}, \quad i=0,1 .
$$

Therefore the operator $U$ satisfies the hypothesis of the conjecture, and hence the inequality (5) holds if the conjecture is true.

On the other hand, if $T$ is an operator as in the conjecture we have

$$
\left\|T U^{-1} f\right\|_{q_{i}, \infty, w} \leq C_{i}\|f\|_{p_{i}, v^{\beta}}, \quad i=0,1,
$$

and by Theorem 1, we obtain

$$
\left\|T U^{-1} f\right\|_{q_{t}, s, w} \leq C_{t, s}\|f\|_{p_{t}, s, v^{\beta}},
$$

for all $0<t<1$ and $1 \leq s \leq \infty$. This inequality together with (5) gives

$$
\|T f\|_{q_{t}, s, w} \leq C_{t, s}\|U f\|_{p_{t}, s, v^{\beta}} \leq C_{t, s}^{\prime}\|f\|_{p_{t}, s, v^{r_{t}}},
$$

completing the proof of the lemma.

Lemma 2. Assume $p_{0}<p_{1}$ and $r_{1}<r_{0}$. There exists a positive weight function $v$ such that (5) is not valid except when $s=p_{t}, 0<t<1$. 
Proof. Let us first observe that if $f$ is a simple function, $f=\sum_{j=1}^{m} c_{j} \chi_{A_{j}}$ with $c_{1}>c_{2}>\ldots c_{m}>0, w\left(A_{j}\right)>0$, and $A_{j} \cap A_{k}=\emptyset$ if $j \neq k$, then we have

$$
\|f\|_{p, s, w}=\left\{\sum_{j=1}^{m} c_{j}^{s}\left(d_{j}^{s / p}-d_{j-1}^{s / p}\right)\right\}^{1 / s},
$$

where $d_{j}=w\left(A_{1}\right)+\cdots+w\left(A_{j}\right), 1 \leq j \leq m$, and $d_{0}=0$. (See e.g. [S-W2], p. 193).

Let us take $p=p_{t}, r=r_{t}, \alpha$ and $\beta$ as in Lemma 1. We define the weight

$$
v(x)= \begin{cases}\sum_{j=1}^{\infty} a_{j} \chi_{E_{j}}, & x \in \bigcup_{j=1}^{\infty} E_{j}, \\ 1, & x \notin \bigcup_{j=1}^{\infty} E_{j},\end{cases}
$$

where $a_{j}=2^{(j-1) /(\beta-r)}, E_{j}=\left\{\left(x_{1}, \ldots, x_{n}\right) \in \Re^{n}: 2^{(j-1) \gamma} \leq x_{i}<2^{j \gamma} \forall i=1, \ldots, n\right\}$ and $\gamma \geq 1$. As a side remark, note that $v(x) \sim|x|^{1 / \gamma(\beta-r)}$ for $x \in \bigcup_{j=1}^{\infty} E_{j}$.

Case $1.1 \leq s<p$. For each $m \in \mathbf{N}$ we can find a set $F_{m} \subset \bigcup_{j=1}^{m} E_{j}$ such that $\left|F_{m} \cap E_{j}\right|=\frac{1}{m a_{j}^{r}}$ for all $1 \leq j \leq m$. This is possible because $r_{1}<r<r_{0}<\beta$, and since $\gamma \geq 1, \frac{1}{m a_{j}^{r}} \leq 1 \leq\left|E_{j}\right|, 1 \leq j \leq m$. Hence,

$$
\left\|\chi_{F_{m}}\right\|_{p, s, v^{r}}=\left(\int_{F_{m}} v^{r}\right)^{1 / p}=\left(\sum_{j=1}^{m} a_{j}^{r}\left|E_{j} \cap F_{m}\right|\right)^{1 / p}=1 .
$$

On the other hand, since

$$
U\left(\chi_{F_{m}}\right)=v^{\alpha} \chi_{F_{m}}=\sum_{j=1}^{m} a_{j}^{\alpha} \chi_{E_{j} \cap F_{m}},
$$

and $\alpha<0$, by (6) we obtain that

$$
\left\|U\left(\chi_{F_{m}}\right)\right\|_{p, s, v^{\beta}}=\left\{\left(\frac{1}{m}\right)^{s / p}+\sum_{j=2}^{m}\left[\left(b_{j}+\frac{1}{m}\right)^{s / p}-b_{j}^{s / p}\right]\right\}^{1 / s}=I,
$$

with $b_{j}=a_{j}^{\alpha p}\left(a_{1}^{\beta}\left|E_{1} \cap F_{m}\right|+\cdots+a_{j-1}^{\beta}\left|E_{j-1} \cap F_{m}\right|\right)=\frac{1}{m} \sum_{k=1}^{j-1} 2^{-k}<\frac{1}{m}$, for all $2 \leq j \leq m$, where we have used $\frac{\alpha p}{\beta-r}=-1$ to prove the second equality. Thus,

$$
\left(b_{j}+\frac{1}{m}\right)^{s / p}-b_{j}^{s / p}>\left(2 b_{j}\right)^{s / p}-b_{j}^{s / p}=\left(2^{s / p}-1\right) b_{j}^{s / p} \geq C \frac{1}{m^{s / p}} .
$$


Then, we can find a constant $C>0$ which does not depend on $m$ such that

$$
I>C\left[m\left(\frac{1}{m}\right)^{s / p}\right]^{1 / s}=C m^{(1 / s)-(1 / p)} .
$$

Since $1 / s-1 / p>0$, the operator $U$ is not bounded from $L^{p, s}\left(v^{r}\right)$ into $L^{p, s}\left(v^{\beta}\right)$.

Case 2. $p<s \leq \infty$. Using (3) and a Hölder-type inequality for Lorentz spaces, we see that (5) is equivalent to

$$
\left\|v^{\alpha+\beta-r} g\right\|_{p^{\prime}, s^{\prime}, v^{r}} \leq C^{\prime}\|g\|_{p^{\prime}, s^{\prime}, v^{\beta}}, \quad \text { for all } g .
$$

To contradict (7) we proceed rather similarly to the previous case, since $s>p$ implies $s^{\prime}<p^{\prime}$. For each $m \in \mathbf{N}$ we can choose a set $G_{m} \subset \bigcup_{j=1}^{m} E_{j}$ such that $\left|G_{m} \cap E_{j}\right|=\frac{1}{m a_{j}^{\beta}}$ for all $1 \leq j \leq m$. Therefore, we can show that

$$
\left\|\chi_{G_{m}}\right\|_{p^{\prime}, s^{\prime}, v^{\beta}}=1
$$

and

$$
\left\|v^{\alpha+\beta-r} \chi_{G_{m}}\right\|_{p^{\prime}, s^{\prime}, v^{r}}>C m^{\left(1 / s^{\prime}\right)-\left(1 / p^{\prime}\right)}
$$

and $U$ turns out to be unbounded again. Hence, the lemma follows.

\section{ACKNOWLEDGEMENT}

The author wishes to thank Professor Eleonor Harboure for her encouragement and guidance.

\section{REFERENCES}

[B-S] C. Bennett and R. Sharpley, Interpolation of operators, Pure and Applied Math. 129, Academic Press, 1988. MR 89e:46001

[C-H-K] H.M. Chung, R.A. Hunt and D.S. Kurtz, The Hardy-Littlewood maximal function on $L(p, q)$ spaces with weights, Indiana Univ. Math. J. 31 (1982), 109-120. MR 83b:42021

[F] E. Ferreyra, Ph.D. Thesis, FAMAF, Univ. Nac. de Córdoba, Córdoba, 1990.

$[\mathrm{M}-\mathrm{W}]$ B. Muckenhoupt and R.L. Wheeden, Weighted norm inequalities for fractional integrals, Trans. Amer. Math. Soc. 192 (1974), 261-274. MR 49:5275

[O] E. Oklander, Interpolación, espacios de Lorentz y teorema de Marcinkiewicz, Cursos y seminarios de matemática, Univ. de Buenos Aires, fasc. 20 (1965). MR 36:680

[Sa] E. Sawyer, Weighted Lebesgue and Lorentz norm inequalities for the Hardy operator, Trans. Amer. Math. Soc. 281 (1984), 329-337. MR 85f:26013

[S-W1] E.M. Stein and G. Weiss, Interpolation of operators with change of measures, Trans. Amer. Math. Soc. 87 (1958), 159-172. MR 19:1184d

[S-W2] E.M. Stein and G. Weiss, Introduction to Fourier analysis on Euclidean spaces, Princeton Univ. Press, 1971. MR 46:4102

Fa.M.A.F., Universidad Nacional de Córdoba, Ciudad Universitaria, 5000 - Córdoba, Argentina

E-mail address: eferrey@mate.uncor.edu 